parent journal, we shall place high priority on rapid editorial decisions and publication. We shall strive for accessibility to a broad readership, and in particular we hope that our News and Views section, which is closely modeled on Nature's, will help to achieve this goal. We shall also be editorially independent in the sense of having no external editorial board. Instead, editorial decisions are made by our full-time editorial staff, consulting with outside experts as we see fit. This is a longstanding policy for both Nature and the monthly Nature titles; it has served us well over the years, allowing editorial flexibility and ensuring that the opinions of a particular individual or group do not dominate editorial policy in any given field.

Despite sharing much in common, the two journals are editorially independent of each other. Thus, it will be for the authors alone to decide where to submit their manuscripts, and Nature will not divert papers to Nature Neuroscience unless the authors explicitly request this. Nature itself will continue to cover the most significant advances in neuroscience as it has always done, and it will judge the manuscripts it receives on their merits, without the involvement of Nature Neuroscience. If, however, a paper cannot be accepted by Nature, authors are welcome to resubmit to Nature Neuroscience. Nature will then release the referees' comments to the editors of Nature Neuroscience, thereby facilitating a rapid editorial decision.

This first issue provides an illustration of the diversity we hope to achieve. The Research Articles cover the spectrum from molecules to cognition, and are arranged in roughly ascending order from low-level to high-level questions. The Scientific Correspondence section (which is fully peer-reviewed) is intended for shorter items that are nevertheless of broad interest to the neuroscience community. The News and Views section is intended to highlight a particular paper (or group of papers on a single theme) and to put the findings in a broader context for non-specialist readers. Most will be devoted to papers appearing in Nature Neuroscience itself, but some (see for instance page 8) will focus on papers of exceptional significance that appear elsewhere. We welcome suggestions for such unlinked pieces, and authors need not be shy about alerting us to their own forthcoming publications in other journals. We also plan to publish reviews on a regular basis, and again we shall be happy to consider suggestions and offers. In the near future, we plan to add Book Reviews and a section for Letters to the editor; the latter may be on matters arising from our previous publications, or on any other topic of interest to our readership.

The problems that neuroscience addresses are almost unique in their scope, and the implications for society are similarly broad. Not only does it hold the promise of new treatments for mental illness, drug addiction, neurodegeneration, cognitive decline with age; neuroscience can also speak-as yet, tentatively-to issues such as education, crime, personality, and to the interplay between cultural environment and biological nature that gives rise to our own mental lives. And finally there is the question of how we came to be here at all; how evolution, that is natural selection acting on sequences of DNA, gave rise to awareness, emotion, intelligence. These are grand themes, and it is easy to forget them when preoccupied with planning the next experiment, writing the next funding application, or for that matter preparing the next issue of a journal. But we should nevertheless remind ourselves occasionally that it is the promise of illuminating such questions that makes the nervous system a uniquely fascinating object of study. Nature Neuroscience looks forward to capturing some of that fascination in the months and years ahead.

Finally, Nature Neuroscience will be published both on paper and electronically; those who are reading this in print are invited to visit our web site (http://neurosci.nature.com), where the text of this entire issue will be freely available from 1 May. We welcome your feedback, and hope that you will find Nature Neuroscience an interesting and valuable source of information.

\section{Into orbit}

On 16 April, the space shuttle Columbia is scheduled to launch into orbit, carrying a cargo of experiments designed to investigate the effect of zero gravity on the nervous system. This mission, code-named Neurolab, is regarded by NASA as one of the pinnacles of its space science program, and neuroscientists may take pride in the fact that NASA finds their research of such interest. But this response must inevitably be tempered by considerations of cost; experiments in space do not come cheap. The cost of a shuttle flight is around half a billion dollars, to which must be added the $\$ 99$ million cost of the mission itself. By this reckoning, these are by far the most expensive neurobiological experiments ever conducted; for comparison, the total 1998 budget for NIMH is $\$ 750$ million. Of course, at one level, such a comparison is misleading. The funding for the Neurolab mission comes from NASA, which allocates less than $2 \%$ of its $\$ 13$ billion budget to life science research. Space science experiments do not compete directly with other biomedical projects; the budget reflects decisions at a much earlier stage in the funding allocation process, when NASA as a whole competes with many other claimants for government R\&D money. In any case, the aims of the space program go far beyond basic scientific research, and nobody would argue that the sole purpose of the space shuttle is to perform basic science in zero gravity.

Nevertheless, basic science is one of NASA's stated aims, and one of its justifications both for the space shuttle and for the forthcoming international space station (on which construction is due to begin this summer). Much of the scientific community has been understandably skeptical; members of the American Society for Cell Biology, for instance, have actively opposed the space station on the grounds that it represents an enormous expenditure with little prospect of serious scientific benefit. The space science program has sometimes been criticized in the past for conducting mediocre research that would not fare well in the earth-based peer review process. But Neurolab has attracted a number of prominent researchers with excellent scientific credentials, and some of the questions they are addressing are of genuine scientific interest; moreover, as one of the investigators, Bruce McNaughton of the University of Arizona, points out, the Neurolab mission has captured the interest of millions of schoolchildren around the country, and the value of this PR to both NASA and the biomedical research community is incalculable. Three projects that illustrate the range of experiments are described in this issue (p 10), and a full list may be found on NASA's own web site (http://neurolab.jsc.nasa.gov).

The results of the Neurolab experiments will appear in NASA technical reports, and investigators are then free to publish them as they see fit. Whether there will be any further follow-up remains to be determined. It may be years before the space station is operational, so many space scientists are hoping for an opportunity in the meantime to extend their projects on another shuttle flight. Whether this will happen is still uncertain; but it behooves the neuroscience community to watch the outcome with interest, since they are as well placed as anyone to judge its scientific value. 